Un nouveau type de plasma, dit ultra-froid,

a récemment été

réalisé en laboratoire

en utilisant des

collisions entre atomes

excités dans un état de

Rydberg, ou en ionisant,

grâce à un laser, un gaz

à une température

proche du zéro absolu.

Les chercheurs essaient

actuellement de

refroidir ces plasmas

et de les piéger tout

en étudiant leurs

propriétés particulières,

comme une possible

cristallisation

spontanée en régime

fortement corrélé.

Figure 1. Classification des plasmas naturels ou de laboratoire, repérés par leur température en Kelvin et par leur densité exprimée en nombre de particules chargées par mètre cube. Dans la zone foncée le plasma est dit cinétique $(\Gamma<1)$, tandis que dans la zone claire le plasma est dit fortement corrélé $(\Gamma \geq 1)$ ( $\Gamma$ est le rapport de l'énergie de Coulomb à l'énergie thermique).

\title{
Aux frontières des plasmas: les plasmas ultra-froids
}

Daniel Comparat (Daniel.Comparat@lac.u-psud.fr), Amodsen Chotia, Matthieu Viteau, Thibault Vogt et Pierre Pillet

Laboratoire Aimé Cotton, CNRS, Univ. Paris-Sud, Bâtiment 505, 91405 Orsay Cedex

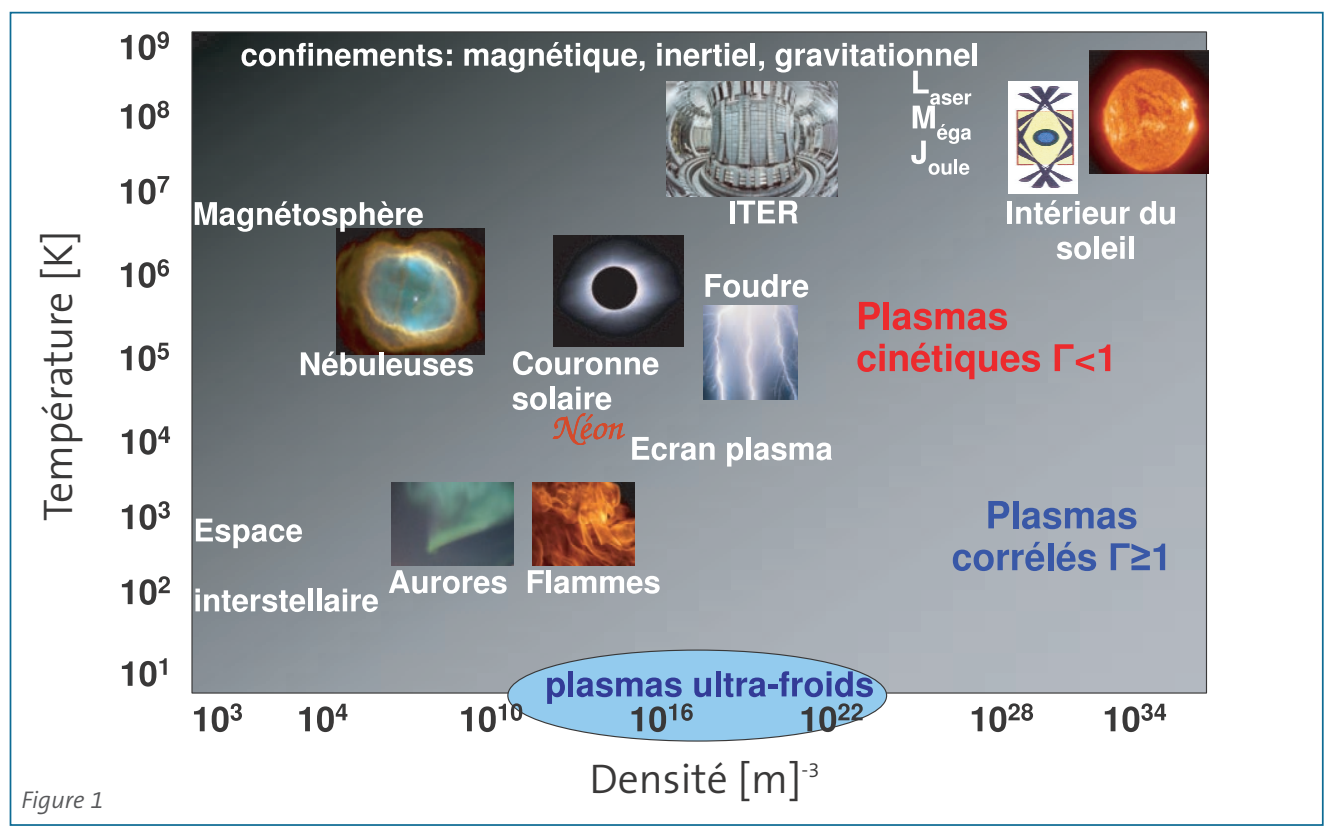

La définition du mot " plasma ", introduit en 1928 par le physicien américain Irving Langmuir, en raison d'une analogie visuelle avec le plasma sanguin, a évolué : elle est passée d'un gaz entièrement ionisé globalement neutre à des systèmes plus complexes, recouvrant un ensemble de particules chargées et parfois excitées, incapables de se recombiner entièrement de manière stable. L'état plasma, qui représente près de $99 \%$ de la matière dans l'univers visible, se rencontre principalement dans les étoiles et le milieu interstellaire. Un plasma provient généralement de collisions très énergétiques qui ionisent les composants atomiques ou moléculaires d'un gaz chauffé à plusieurs milliers de degrés. Ceci explique pourquoi l'état plasma est pratiquement inexistant sur terre, hormis lors d'éclairs ou d'aurores boréales. De façon intéressante, un plasma peut toutefois se former à basse température si la source d'ionisation lui est extérieure. C'est le cas de l'ionosphère qui, bien que froide, subit en permanence un intense bombardement ionisant de particules venant du Soleil, se manifestant par exemple lors des aurores polaires.

Dans cet article, nous considérerons le cas fréquent des plasmas neutres et simplement ionisés. Les densités moyennes des ions et des électrons, $\mathrm{n}_{\mathrm{i}}$ et $\mathrm{n}_{\mathrm{e}}$, sont alors égales : $\mathrm{n}_{\mathrm{i}}=\mathrm{n}_{\mathrm{e}}$. Ce type de plasma est caractérisé par la force des interactions mises en jeu dépendant de la température $\mathrm{T}_{\mathrm{e}}$ et de la densité $\mathrm{n}_{\mathrm{e}}$ des électrons. La température $T_{e}$ permet de connaitre l'énergie cinétique $\mathrm{E}_{\mathrm{cin}} \sim \mathrm{k}_{\mathrm{B}} \mathrm{T}_{\mathrm{e}}$ d'un électron, alors que la densité $\mathrm{n}_{\mathrm{e}}$ est reliée à l'interaction potentielle coulombienne $\mathrm{E}_{\text {pot }} \sim \mathrm{e}^{2} / \mathrm{a}$ entre deux électrons ( $\mathrm{e}^{2}=\mathrm{q}_{\mathrm{e}}{ }^{2} / 4 \pi \varepsilon_{0}$ où $\mathrm{q}_{\mathrm{e}}$ est la charge de l'électron), dépendant essentiellement de la distance moyenne entre plus proches voisins $\mathrm{a}=\left(4 \pi \mathrm{n}_{\mathrm{e}} / 3\right)^{-1 / 3} . \mathrm{T}_{\mathrm{e}}$ et $\mathrm{n}_{\mathrm{e}}$ sont donc les deux paramètres à la base de la classification des plasmas présentée sur la figure 1.

Le but de cet article est de présenter un nouveau type de plasma à la frontière de cette classification. Des expériences récentes permettent en effet de créer des plasmas " ultra-froids " par ionisation d'un gaz de température initiale inférieure au milli-Kelvin.

Les plasmas que nous allons étudier se situent dans la partie basse température de cette classification et permettent donc d'aborder un nouveau type de physique. En particulier, ils permettent d'approcher en laboratoire un état "fortement corrélé » où l'énergie de Coulomb domine l'énergie thermique. Dans ce type de plasma, le paramètre plasma $\Gamma=\mathrm{E}_{\text {pot }} / \mathrm{E}_{\text {cin }}\left(\Gamma_{\mathrm{e}}\right.$ pour les électrons et $\Gamma_{\text {ion }}$ pour les ions), qui compare l'énergie de Coulomb et l'énergie thermique, devient plus grand que l'unité. De par la nature même de la formation d'un plasma - ionisations lors de collisions suffisamment 
énergétiques - cette situation corrélée n'est pratiquement jamais réalisée dans un plasma naturel [1]. Indiquons dès à présent qu'un tel état (plasma neutre corrélé) n'a pas encore été réalisé en laboratoire ; mais les effets physiques attendus sont particulièrement spectaculaires, comme le représente la modélisation (effectuée à Dresde en Allemagne) de la figure 2b. La théorie prédit des corrélations en positions des électrons ou des ions pour $\Gamma>1$ et une cristallisation, i.e. un ordre à très grande distance, si $\Gamma>174$. Ceci serait très similaire à ce qui a été expérimentalement observé dans le cas d'un plasma non neutre (figure 2a) formé uniquement d'ions piégés dans un piège électrostatique et refroidis par laser, jusqu'à ce que l'énergie potentielle domine largement l'énergie cinétique $\left(\Gamma_{\text {ion }}>300\right)$. L'image, observée par diffraction de Bragg lumineuse dans une expérience au NIST (États-Unis), montre clairement que le système tend à se mettre dans une configuration d'énergie minimale correspondant à des ions éloignés le plus possible de leurs voisins, formant alors un réseau (cristal de Wigner). Les recherches que nous allons décrire portent sur des plasmas neutres qui semblent encore plus intéressants à étudier, car l'effet de l'interaction coulombienne est moins évident que dans le cas d'un plasma non neutre, les charges ioniques étant alors écrantées par la présence d'électrons.

\section{Les plasmas ultra-froids}

L'une des premières tentatives pour atteindre l'état de plasma "ultra-froid " a eu lieu à Orsay dans les années 1970 et consista à ioniser de l'hélium superfluide à la température d'environ $4 \mathrm{~K}$ [2] en lui appliquant une impulsion de champ électrique. Malheureusement, ce plasma, dit cryogénique, n'est pas à l'équilibre thermodynamique et des recombinaisons à trois corps électrons-ions chauffent les électrons restants (voir encadré p. 12), empêchant l'observation du régime corrélé.

Mais les laboratoires disposent depuis plus d'une quinzaine d'années de gaz bien plus froids que ces températures cryogéniques : ce sont les gaz d'atomes refroidis par laser avec typiquement $\mathrm{N}>10^{6}$ atomes, une densité $\mathrm{n} \sim 10^{17} \mathrm{~m}^{-3}$ et une température $\mathrm{T} \sim 100 \mu \mathrm{K}$. Il semble naturel de penser qu'en partant d'une température plus basse, le plasma final sera lui aussi plus froid et approchera peut-être le régime corrélé. De façon étrange, il fallut attendre plus d'une dizaine d'années avant que les physiciens aient ainsi la volonté d'ioniser des nuages d'atomes refroidis par laser pour former des plasmas dits « ultra-froids ». L'idée n'a, finalement, pas émergé de la communauté des "plasmiciens " mais de celle des physiciens atomistes, pour qui ces plasmas étaient tout d'abord considérés nocifs à leurs mesures, comme par exemple celles des sections efficaces d'ionisation.

Le premier plasma ultra-froid ne fut donc réalisé qu'en 1999 aux États-Unis (laboratoire NIST à Gaithersburg) en photoionisant des atomes de xénon métastables [3]. Depuis, une dizaine de groupes de par le monde étudient ces plasmas en partant d'atomes différents : rubidium, césium, strontium ou béryllium. En choisissant la longueur d'onde du laser, il semble a priori possible de choisir la température électronique du plasma. En effet, à cause de la très faible masse de l'électron comparée à celle de l'ion, la différence entre l'énergie du photon et l'énergie d'ionisation de l'atome est presque entièrement transférée aux électrons sous forme d'énergie cinétique. Sur la figure $2 \mathrm{~b}$ est représenté le plasma (visiblement très corrélé car cristallisé) que l'on pensait à l'époque naïvement possible de réaliser en ionisant «au seuil » avec presque aucune énergie cinétique : $\mathrm{n}_{\mathrm{e}} \sim \mathrm{n}_{\mathrm{i}} \sim 10^{16} \mathrm{~m}^{-3}$ et $\mathrm{T}_{\mathrm{e}} \sim \mathrm{T}_{\mathrm{i}} \sim 100 \mu \mathrm{K}$. Mais, comme pour les plasmas cryogéniques, on s'aperçut rapidement que la recombinaison à trois corps entraîne un chauffage rapide des électrons, élevant leur température à $\mathrm{T}_{\mathrm{e}} \sim 10 \mathrm{~K}$ et éloignant le système du régime corrélé.

La figure 3 résume l'évolution d'un plasma ultra-froid après l'ionisation des atomes. Les électrons, qui sont plus légers et qui se meuvent plus rapidement que les ions, quittent en premier la zone d'ionisation jusqu'à ce que le système soit très légèrement chargé positivement et que

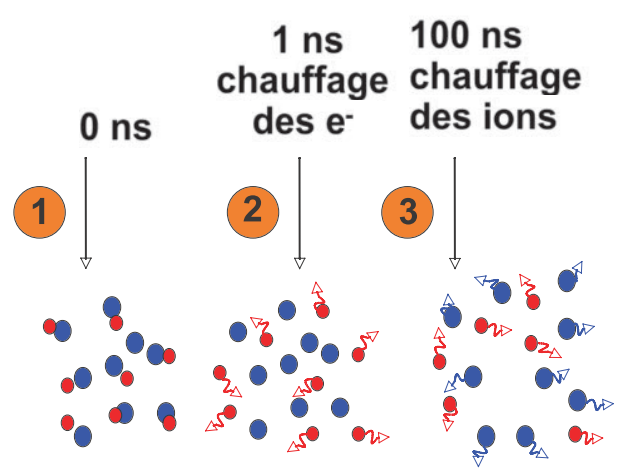

$1 \mu \mathrm{s}$

chauffage des $\mathrm{e}^{-}$

par recombinaison Expansion à trois corps

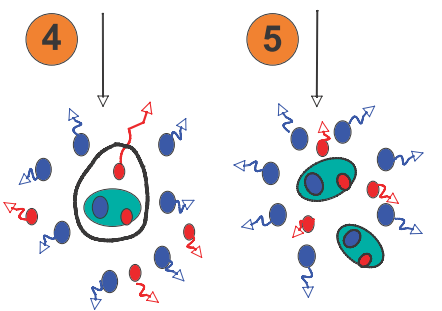

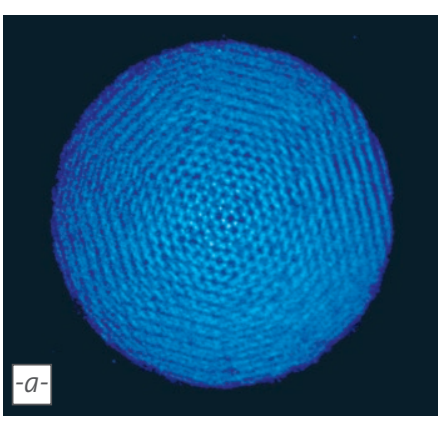
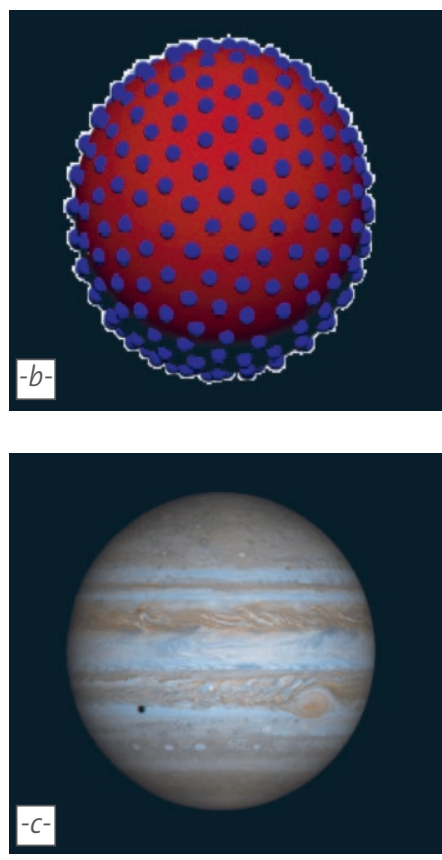

Figure 2. Exemples de plasmas fortement corrélés. a) Plasma ultra-froid non neutre d'ions $\mathrm{Be}^{+}$dans un piège à ions, $T_{i}<5 \mathrm{mK}$, $\left.n_{i}=10^{14} \mathrm{~m}^{-3}, \Gamma_{i o n}>300 . b\right)$ Modélisation d'un plasma ultra-froid neutre avec $T_{e} \sim T_{i} \sim 100 \mu \mathrm{K}, \Gamma_{\text {ion }}>174$. Les ions sont représentés en bleu et les électrons en rouge. c) Jupiter contiendrait des plasmas fortement corrélés en son centre, tout comme les étoiles à neutrons ou les naines blanches.
Figure 3. Évolution temporelle d'un plasma ultra-froid après sa création (1). 2) Juste après l'ionisation ( 1 ns), des électrons (cercles rouges) quittent la zone d'ionisation. 3) Mouvement et chauffage dus à la répulsion entre ions (cercles bleus) induite par leur désordre initial. 4) Recombinaison des ions et des électrons formant des atomes excités. 5) Expansion amenant peu à peu à la mort du plasma. 


\section{Recombinaisons à trois corps et atomes de Rydberg}

Dans un plasma formé, par exemple, d'électrons $\mathrm{e}^{-}$et d'ions $\mathrm{M}^{+}$, des collisions entre deux électrons et un ion (on parle de recombinaison à trois corps) peuvent mener à une recombinaison électron-ion formant un atome neutre via la réaction : $\mathrm{e}^{-}+\mathrm{e}^{-}+\mathrm{M}^{+} \rightarrow \mathrm{M}+\mathrm{e}^{-}$.

Par conservation de l'énergie, l'électron libre emporte l'énergie et l'impulsion libérées par la formation de la paire. Cette réaction se produit à un taux proportionnel à $T_{e}^{-4,5}$, ce qui explique pourquoi elle devient un élément prépondérant de la physique des plasmas ayant une basse température électronique $T_{e}$.

Les atomes $M$ ainsi formés sont généralement dans un état très excité ; l'électron n'est que très peu lié au cœur ionique $M^{+}$: on parle d'atomes de Rydberg (grand nombre quantique principal n). La caractéristique principale de tels atomes est leur taille énorme (pouvant être de l'ordre du micromètre) et leur fragilité. Un faible champ électrique ou bien une collision peu énergétique peuvent par exemple amener à leur ionisation. Dans ce dernier cas, l'ionisation s'effectue via la réaction inverse de la recombinaison à trois corps précédemment décrite.

Atome de Rydberg

Figure 4. Haut : Expérience présentant le nombre d'électrons du plasma en fonction du temps d'évolution d'un gaz d'atomes de Rydberg ultra-froid. Bas : Schéma de l'évolution d'un tel gaz; les courbes représentent schématiquement des puits de potentiel. 1) Ionisation via le rayonnement $d u$ corps noir ou via des collisions induites par les forces de type Van der Waals. 2) Collision des électrons piégés avec les atomes restants. Une réaction en chaîne se produit : elle change l'état interne des atomes et ionise rapidement une grande partie de l'ensemble des atomes présents. 3) Expansion du plasma.
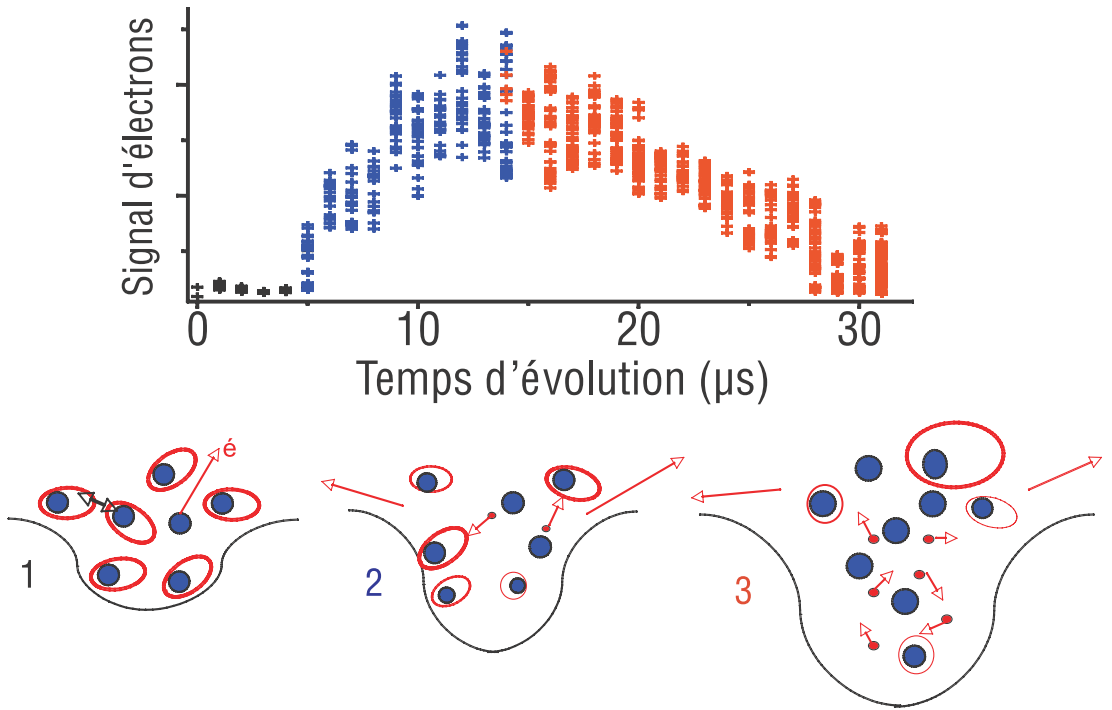

de l'inverse de la pulsation plasma ionique $\omega_{\mathrm{i}}=\left(\mathrm{n}_{\mathrm{i}} \mathrm{e}^{2} / \mathrm{m}_{\mathrm{i}} \varepsilon_{0}\right)^{1 / 2}$. La pression du gaz d'électrons amène enfin à l'expansion quasiment autosimilaire (conservant la forme gaussienne) du plasma et à sa dilution au cours des quelques dizaines de microsecondes de vie de celui-ci. Le nuage s'étend enfin sous l'effet de la pression des électrons.

\section{Diagnostics expérimentaux}

La technique de diagnostic privilégiée des plasmas ultra-froids est la spectroscopie d'absorption. Cette méthode convient aux ions (béryllium, strontium...) ayant encore un électron de valence, mais non aux ions alcalins (rubidium, césium...) ou de gaz rare (xénon). Un faisceau laser de longueur d'onde correspondant à la transition de l'ion et traversant le plasma donne sur une caméra CCD l'image de la densité $\mathrm{n}_{\mathrm{i}}$ des ions absorbant la lumière. L'élargissement en fréquence lié à l'effet Doppler, créé par le mouvement des ions, permet de déduire la température $\mathrm{T}_{\mathrm{i}}$. En étudiant la taille du nuage en fonction du temps, on en déduit la vitesse d'expansion radiale des ions $\mathrm{V}_{\text {rad. }}$ La pression des électrons agit sur les ions et un transfert d'énergie cinétique intervient, conduisant à l'expression $\mathrm{V}_{\mathrm{rad}}=\left(\mathrm{k}_{\mathrm{B}} \mathrm{T}_{\mathrm{e}} / \mathrm{m}_{\mathrm{i}}\right)^{1 / 2}$. Cela permet d'avoir accès aux premiers paramètres $n_{i}$ et $T_{i}$, et de manière un peu indirecte à $\mathrm{T}_{\mathrm{e}}$.

Une technique, mise en ouvre en 1999 par l'équipe du NIST et adaptée à tout type d'ion, permet en plus de déterminer la densité électronique $\mathrm{n}_{\mathrm{e}}$ en excitant les électrons du plasma grâce à un petit champ électrique radiofréquence externe. Lorsque cette fréquence atteint la valeur de la pulsation plasma $\omega_{\mathrm{e}}$, le mouvement des électrons est fortement amplifié, les électrons sont chauffés et éjectés du plasma. En mesurant, en fonction de la fréquence, le nombre de ces électrons sur un détecteur placé autour du plasma, on peut connaitre $\omega_{\mathrm{e}}$ et donc $\mathrm{n}_{\mathrm{e}}$. 


\section{Autoionisation d'atomes}

La physique entourant les plasmas ultra-froids fut enrichie en l'an 2000 grâce à l'observation, par notre équipe de recherche, du phénomène inverse de la recombinaison à trois corps [4]. Une assemblée suffisamment dense d'atomes, préalablement refroidis par laser et excités dans un état de Rydberg, s'ionise pour former un plasma ultra-froid. L'équilibre thermodynamique lié à la recombinaison à trois corps explique pourquoi le plasma ainsi formé a finalement des caractéristiques $\left(\mathrm{n}_{\mathrm{i}} \sim \mathrm{n}_{\mathrm{e}} \sim 10^{15} \mathrm{~m}^{-3}, \mathrm{~T}_{\mathrm{e}} \sim 10 \mathrm{~K}\right.$, $\mathrm{T}_{\mathrm{i}} \sim 1 \mathrm{~K}$ ) similaires au plasma formé par laser ; son évolution finale est donc la même : expansion sous l'effet de la pression des électrons.

Cette ionisation sembla à l'époque paradoxale, car l'énergie cinétique pour ioniser ces atomes ultra-lents (car très froids) ne semblait pas exister. L'ignition du processus est en effet complexe et plusieurs phénomènes entrent en jeu. Un atome de Rydberg peut être ionisé en absorbant le rayonnement infrarouge du corps noir provenant des parois de l'enceinte qui sont à température ambiante, ou s'ioniser par collision avec un proche voisin grâce à la vitesse importante créée par les forces attractives entre eux. Le processus (décrit en figure 4) se poursuit alors jusqu'au point où les électrons formés sont piégés par l'excès d'ions créé. Il s'ensuit des collisions entre ces électrons piégés et les atomes restants, modifiant l'état de l'atome en changeant son nombre quantique principal et/ou en changeant le moment angulaire de rotation de son électron. Les atomes de Rydberg peu liés (i.e. dont l'énergie de liaison est inférieure à l'énergie cinétique $\sim \mathrm{k}_{\mathrm{B}} \mathrm{T}_{\mathrm{e}}$ d'un électron libre du plasma) sont ionisés. Les atomes de Rydberg plus liés, quant à eux, voient leur nombre quantique principal diminuer rapidement et vont se désexciter dans leur état fondamental par émission spontanée ; ils deviennent alors spectateurs de l'évolution ultérieure du système.

\section{Modélisation : analogie avec la dynamique des amas globulaires stellaires}

La physique des plasmas est à la frontière de nombreuses disciplines (physique atomique et moléculaire, fluides, statistiques, chimie, astrophysique, électromagnétisme...) et s'est toujours enrichie de leurs approches variées et complémentaires. C'est dans cette optique que notre équipe de recherche a mené une étude utilisant l'analogie, surprenante au premier abord, avec le système formé par un amas d'étoiles [5]. La principale force (coulombienne) intervenant entre

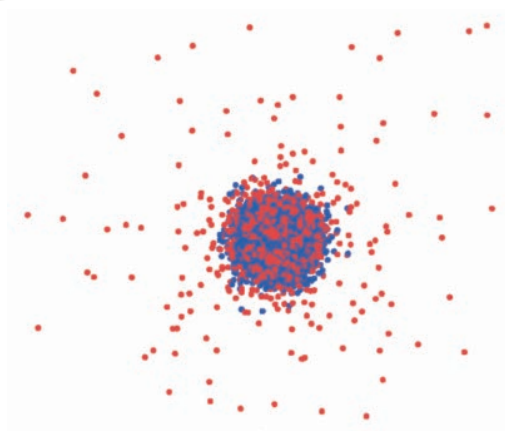

Plasma ultra-froid

•. $\quad F=\left(\mathrm{q}_{\mathrm{e}}{ }^{2} / 4 \pi \varepsilon_{0}\right) / \mathrm{r}^{2}$

deux particules dans le plasma varie en effet comme l'inverse du carré de la distance, tout comme la force newtonienne de la gravitation. L'analogie entre l'évolution des amas globulaires stellaires et les plasmas est en fait assez ancienne car, déjà dans les années 60, Il y a Prigogine dénommait ces amas globulaires des « plasmas gravitationnels ", et la théorie des galaxies comme plasmas gravitationnels est maintenant bien développée.

La figure 5 présente visuellement cette analogie dans le cas d'un plasma ultra-froid. Le potentiel total est globalement attractif dans les deux systèmes. Cela est évident pour les étoiles ; pour les électrons, la présence des ions assure un confinement globalement attractif à l'ensemble, malgré la présence de deux types de charge avec des interactions attractives et répulsives.

Les équations d'évolution des électrons du plasma ou des étoiles de l'amas sont formellement identiques, moyennant le changement de constante de gravitation $\left(\mathrm{G} \leftrightarrow \mathrm{e}^{2} / \mathrm{m}_{\mathrm{e}}^{2}\right)$. Il est donc possible d'utiliser l'important travail réalisé sur les amas globulaires pour étudier la dynamique collisionnelle des électrons de notre plasma ; par exemple, les codes informatiques de simulations numériques sont facilement transposables d'un système à l'autre. De plus, toute particule (électron ou étoile) ayant une énergie supérieure au potentiel global attractif s'en va à l'infini, ce qui permet par exemple de montrer analytiquement que la distribution d'équilibre est une "maxwellienne abaissée " appelée distribution de Kramers-Michie-King.

$\mathrm{La}$ recombinaison à trois corps forme des systèmes binaires, étoiles binaires dans un amas ou atomes de Rydberg dans un plasma, à un taux identique dans les deux systèmes :

$\mathrm{n}_{\text {étoile }}{ }^{2} \mathrm{G}^{5}\left(\mathrm{k}_{\mathrm{B}} \mathrm{T}_{\text {étoile }} / \mathrm{m}_{\text {étoile }}\right)^{-9 / 2}$

ou $n_{e} n_{i}\left(e^{2 / m_{e}}\right)^{5}\left(k_{B} T_{e} / m_{e}\right)^{-9 / 2}[5]$.

Cette analogie permet de noter une fois encore que notre système est un système modèle. Il réalise en un certain sens un amas globulaire idéal en laboratoire car il vérifie, bien mieux que les amas stellaires réels, des hypothèses de sphéricité, de présence d'une seule classe de masse, de particules de durée de vie infinie (pas d'évolution stellaire), souvent utilisées pour déduire les équations d'évolution des amas stellaires.

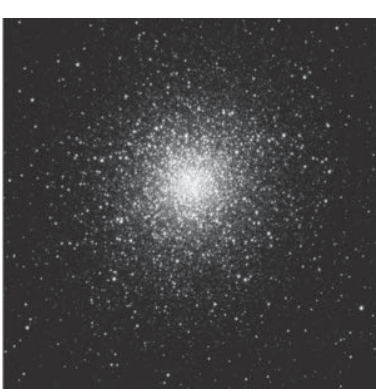

Amas globulaire

$\mathrm{F}=\left(-\mathrm{G} \mathrm{M}^{2}\right) / \mathrm{r}^{2}$

Figure 5. Analogie entre la dynamique d'un plasma ultra-froid (modélisé par une équipe de San Diego, à gauche) et d'un amas stellaire (image de l'amas d'Hercule, à droite). Les deux systèmes présentent un même nombre de particules, une symétrie sphérique, le même type de forces. Les systèmes binaires, étoiles ou atomes excités, sont soumis aux mêmes lois (taux de changement d'énergie de liaison par exemple) vis-à-vis des collisions avec leurs partenaires.

\section{Pour en savoir plus}

[1] S. Ichimaru, "Strongly coupled plasma", Rev. Mod. Phys. 54, 1017 (1982)

[2] J.-F. Delpech et

J.-C. Gauthier, "Electron-Ion Recombination in Cryogenic Helium Plasmas", Phys. Rev. A 6, 1932 (1972).

[3] T.C. Killian et al. "Creation of an Ultracold Neutral Plasma", Phys. Rev. Lett. 83, 4776 (1999).

[4] M.P. Robinson et al., "Spontaneous evolution of Rydberg atoms into an ultracold plasma", Phys. Rev. Lett. 85, 4466 (2000).

[5] D. Comparat et al.

"Star cluster dynamics

in a laboratory: electrons

in ultracold plasma", Mon. Not.

R. Astron. Soc. 361, 1227 (2005).

[6] Topical issue on "Ultracold plasmas and cold Rydberg atoms", Eur. Phys. J. D 40, 1-80 (2006).

[7] PARYS International

Workshop on cold Rydberg

Gases and ultracold Plasmas (2005), www.lac.u-psud.fr/

LAC/PARYS/PARYS4.htm

[8] T. C. Killian et al.,'Ultracold Neutral Plasmas",

arXiv.org:physics/0612097.

[9] B. J. Claessens et al., "Ultracold Electron Source", Phys. Rev. Lett. 95, 164801 (2005). 


\section{Conclusion et perspectives}

Les recherches concernant les plasmas ultra-froids sont en pleine évolution et s'ouvrent vers des domaines très divers allant de l'étude spectroscopique à la recombinaison en atomes de Rydberg, en passant par l'étude des fluctuations et instabilités induites, par exemple, par des champs externes $[6,7,8]$. Au vu de la simplicité théorique et du très bon contrôle expérimental des paramètres $\mathrm{n}_{\mathrm{e}}$ et $\mathrm{T}_{\mathrm{e}}$ via l'intensité et la longueur d'onde laser, un plasma ultra-froid est un système modèle pour l'étude de la plupart des processus (explosion coulombienne, instabilités, excitations collectives, recombinaison...) qui existent dans de nombreux types de plasmas.

Les plasmas ultra-froids ouvrent la porte à plusieurs types d'applications. Sur le plan fondamental, il y a un lien très étroit entre les plasmas ultra-froids et les expériences de formation d'anti-hydrogène lors de recombinaisons à trois corps au sein d'un plasma cryogénique d'antiprotons et de positrons.
Les applications pratiques les plus intéressantes des plasmas ultra-froids concernent les sources ultra-brillantes d'électrons ou d'ions lents. Il suffit pour cela d'extraire, à l'aide d'un champ électrique impulsionnel, les particules chargées présentes dans un plasma ultra-froid. De telles sources d'électrons sont, par exemple, le cœur des lasers à électrons libres ou des systèmes d'imagerie électroniques. On pourrait envisager un microscope électronique avec une résolution temporelle femtoseconde, pour sonder les surfaces chauffées par des lasers ultra-rapides, pour observer des structures en femto-chimie, pour l'étude des transitions de phase en matière condensée... En accélérant ces sources d'électrons à des vitesses relativistes, la diffusion Compton de lumière laser pourrait former des sources compactes de rayons X [9]. Les enjeux sont énormes : ainsi, le projet mené à Eindhoven aux Pays-Bas, porte sur une source $10^{8}$ fois plus brillante qu'un synchrotron avec une résolution temporelle mille fois meilleure ! 